\title{
Commentary \\ EMSY links breast cancer gene 2 to the 'Royal Family'
}

Jun Yao and Kornelia Polyak

\author{
Department of Medical Oncology, Dana-Farber Cancer Institute, Boston, Massachusetts, USA \\ Corresponding author: Kornelia Polyak, Kornelia_Polyak@dfci.harvard.edu \\ Published: 19 July 2004 \\ Breast Cancer Res 2004, 6:201-203 (DOI 10.1186/bcr916) \\ (C) 2004 BioMed Central Ltd
}

\begin{abstract}
Although the role of the breast cancer gene 2 (BRCA2) tumor suppressor gene is well established in inherited breast and ovarian carcinomas, its involvement in sporadic disease is still uncertain. The recent identification of a novel BRCA2 binding protein, EMSY, as a putative oncogene implicates the BRCA2 pathway in sporadic tumors. Furthermore, EMSY's binding to members of the 'Royal Family' of chromatin remodeling proteins may lead to a better understanding of the physiological function of BRCA2 and its role in chromatin remodeling.
\end{abstract}

Keywords: BRCA2, breast cancer, EMSY, familiar, sporadic

Breast cancer, similar to other tumor types, develops as a result of cumulative genetic and epigenetic changes. It is well established that the majority of inherited breast and ovarian carcinomas are due to germline mutations in the breast cancer gene 1 (BRCA1) and breast cancer gene 2 (BRCA2) tumor suppressor genes [1-4], but these inherited cases account for less than $5 \%$ of all breast carcinomas. In the far more common sporadic form of the disease, mutations or deletion of BRCA1 and BRCA2 have not been detected [5]. This contrasts with other tumor suppressors such as TP53 and adenomatous polyposis coli in which both germline and somatic mutations play a role in tumorigenesis.

Although loss of BRCA1 due to promoter hypermethylation and loss of heterozygosity has been reported in a fraction of breast and ovarian carcinomas [6,7], there is presently no report on how BRCA2 might be impaired in sporadic tumors. In a recent issue of Cell, HughesDavies and colleagues described the provocative finding that the EMSY putative oncogene can interact with BRCA2 and may inhibit its function [8]. If this hypothesis proves to be correct, then EMSY amplification would provide a possible explanation for the lack of BRCA2 mutation in sporadic breast cancers and would further underline the importance of BRCA proteins in breast tumorigenesis.

The BRCA2 gene encodes a large (3418 amino acids) protein proposed to function in DNA repair, homologous recombination, chromatin remodeling, and regulation of transcription [9-11]. For instance, BRCA2 is recruited to RAD51-containing nuclear foci after DNA damage, where BRCA2 participates in DNA repair. Consistent with this finding, BRCA2 null cells from human tumors as well as cells from knockout mice are sensitive to ionization radiation [12-15]. When fused to a heterologous DNA binding domain, BRCA2 demonstrated the ability to stimulate transcription [16-18]. However, the physiologic targets regulated by these putative BRCA2 transactivation domains, and the physiologic relevance of this function and its necessity for tumor suppressor activity, are unknown since all of these studies were performed in vitro using overexpressed proteins.

A recent study reported that exogenously expressed BRCA2 can stimulate transcription of androgen receptorregulated genes in human 293 cells [19], but the relevance of this finding in breast tumorigenesis is unclear. The only evidence for the importance of the BRCA2 
putative transactivation domain is a germline mutation in a breast and ovarian cancer family that only removes its putative transactivation domain due to an inframe deletion, although even in this case the possibility of overall structural effects cannot be excluded [20].

In the search for proteins that may interact with the BRCA2 transactivation domain, Hughes-Davies and colleagues performed a yeast two-hybrid screen and identified a novel protein they termed EMSY [8]. The interaction between recombinant and endogenous EMSY and BRCA2 was confirmed using multiple different methods, while the consequences of this interaction were demonstrated in a reporter assay using a BRCA2 transactivation and the GAL4 DNA binding domain fusion protein. Co-expressing EMSY or its $\mathrm{N}$-terminus portion was sufficient to suppress transactivation by the BRCA2-GAL4 fusion protein from a GAL4 promoter. These results indicate that EMSY may conceal the BRCA2 transactivation function through direct binding to its transactivation domain. Based on this observation, the authors immediately raised the question of whether EMSY might function as an oncoprotein that compromises BRCA2 function in sporadic breast and ovarian carcinomas.

Interestingly, based on its intracellular localization, EMSY, similar to BRCA2, is also implicated in DNA damage. Following ionization radiation, Hughes-Davies and colleagues observed the recruitment of EMSY into nuclear foci in a murine fibroblast cell line, although this could not be reproduced in human cells [8]. DNA damage-induced nuclear foci are thought to be composed of proteins involved in DNA damage response and repair that aggregate at the damaged sites to perform repair functions. Among these proteins, gamma histone $2 A X$ is one of the earliest to aggregate into nuclear foci [21], whereas BRCA2 is recruited into the foci at a later time point $[15,22]$. EMSY foci were detected shortly after DNA damage and almost completely colocalized with phosphogamma histone $2 A X$, suggesting a role for EMSY in early response to DNA damage. Many proteins (e.g. gamma histone 2AX, BRCA1, and 53BP1) aggregating into the foci are phosphorylated on S/TQ sites (serine or threonine followed by glutamine) by the ataxia-telangiectasia mutated gene (ATM) kinase in response to DNA damage. Interestingly, EMSY carries similar sites, raising the possibility that EMSY might be a new ATM substrate. Whether EMSY foci require BRCA2 (or vice versa), and if the EMSY-BRCA2 interaction influences BRCA2's binding to RAD51 and recombination-coupled DNA repair, all remain to be determined.

By performing an additional yeast two-hybrid screen using EMSY as bait, Hughes-Davies and colleagues also discovered that EMSY can interact with HP1 and BS69, proteins containing 'Royal Family' domains and implicated in chromatin remodeling [23]. Chromatin remodeling is yet another cellular process in which BRCA2 has been implicated $[24,25]$. Taken together, these data imply that EMSY may be involved in many cellular functions in which BRCA2 is thought to play a role, suggesting that the EMSY-BRCA2 interaction may have physiologic relevance and may influence the tumor suppressor function of BRCA2.

The localization of the EMSY gene to chromosome $11 q 13$, a region amplified in $\sim 13 \%$ of breast carcinomas $[26,27]$, provided a clue that EMSY may play a role in sporadic tumors by acting as an oncogene. In order to test this hypothesis, Hughes-Davies and colleagues examined EMSY's copy number and expression in a panel of breast cancer cell lines. Using real-time PCR, array comparative genomic hybridization, and fluorescence in situ hybridization, they found that EMSY is overexpressed and amplified in a subset of breast cancer cell lines. Similarly, they detected EMSY amplification in 7.4-13\% of sporadic breast tumors, but the expression of EMSY in primary breast tumors was not analyzed.

Moreover, the EMSY copy number is inversely correlated with survival in a subset of breast cancer patients. Specifically, the median disease-free survival of patients with lymph node-negative tumors with EMSY amplification was 6.4 years versus that of 14 years in cases without EMSY amplification. This suggests that EMSY amplification may have prognostic value in breast cancer, although the tumor size was still a stronger predictor of outcome than EMSY amplification and tumor grade was not analyzed. Moreover, since most amplicons are large and include many genes, it is notoriously hard to discriminate the target of an amplicon from genes that just 'go along for the ride'. This is especially true for the gene-rich $11 \mathrm{q} 13$ amplicon that includes several proven oncogenes like cyclin D1, glycoprotein a repetitions predominant gene (GARP), and PAK1. Hughes-Davies and colleagues had two pieces of evidence to argue that EMSY might be one of the potential targets of the 11q13 amplicon, at least in a subset of breast tumors. First, using quantitative real-time PCR analysis, they demonstrated that while GARP and EMSY are co-amplified in breast cancer cell lines, only EMSY appears to be overexpressed in cells with 11 q13 copy number gain. Second, GARP/EMSY amplification is detected in $7-13 \%$ of sporadic breast cancers, and in $3-5 \%$ of breast tumors GARP/EMSY amplification is not associated with that of cyclin $D_{1}$.

\section{Conclusions}

Despite compelling evidence, the crucial question of whether EMSY is a bona fide surrogate for BRCA2 mutation in sporadic cancers still remains to be answered. Due to the fact that several other BRCA2 interacting 
proteins are mutated or overexpressed in breast cancer (BubR1, BCCIP alpha, ATM, and Plk1), the EMSY-BRCA2 interaction can only be one part of the story. Although EMSY is implicated in many aspects of BRCA2 function, more work is required to strengthen these putative links. For example, it would be important to know whether breast cancer cell lines with EMSY amplification display some of the phenotypes (radiosensitivity and deficiency in homologous recombination) characteristic of BRCA2 null cells. More evidence is also needed to prove that EMSY is overexpressed and amplified in primary breast and ovarian carcinomas. Moreover, it would be important to know in which breast cancer subtypes EMSY amplification occurs and what is the status of EMSY in BRCA2 null tumors. Experimental models in cell lines and transgenic mice are desired to prove the functional relevance of EMSY amplification and overexpression, and whether it is a target of the $11 \mathrm{q} 13$ amplicon.

Nevertheless, with a new partner at hand, the BRCA2 field is ready for a new dance and members of the 'Royal Family' are invited to join ...

\section{Competing interests}

None declared.

\section{Acknowledgements}

The authors would like to thank Dr Dale Porter and Ms Annette März for critical reading of the manuscript. Supported in part by the SPORE in Breast Cancer at Dana-Farber/Harvard Cancer Center.

\section{References}

1. Miki Y, Swensen J, Shattuck-Eidens D, Futreal PA, Harshman K, Tavtigian S, Liu Q, Cochran C, Bennett LM, Ding W, et al.: A strong candidate for the breast and ovarian cancer susceptibility gene BRCA1. Science 1994, 266:66-71.

2. Wooster R, Stratton MR: Breast cancer susceptibility: a complex disease unravels. Trends Genet 1995, 11:3-5.

3. Hofmann W, Schlag PM: BRCA1 and BRCA2 - breast cancer susceptibility genes. J Cancer Res Clin Oncol 2000, 126:487496.

4. Paakkonen K, Sauramo S, Sarantaus L, Vahteristo P, Hartikainen $A$, Vehmanen $\mathrm{P}$, Ignatius J, Ollikainen $\mathrm{V}$, Kaariainen $\mathrm{H}$, Vauramo $\mathrm{E}$, et al:: Involvement of BRCA1 and BRCA2 in breast cancer in a western Finnish sub-population. Genet Epidemiol 2001, 20: 239-246.

5. Gayther SA, Pharoah PD, Ponder BA: The genetics of inherited breast cancer. J Mammary Gland Biol Neoplasia 1998, 3:365376.

6. Dobrovic A, Simpfendorfer D: Methylation of the BRCA1 gene in sporadic breast cancer. Cancer Res 1997, 57:3347-3350.

7. Wilson CA, Ramos L, Villasenor MR, Anders KH, Press MF, Clarke K, Karlan B, Chen JJ, Scully R, Livingston D, et al:: Localization of human BRCA1 and its loss in high-grade, non-inherited breast carcinomas. Nat Genet 1999, 21:236-240.

8. Hughes-Davies L, Huntsman D, Ruas M, Fuks F, Bye J, Chin SF, Milner J, Brown LA, Hsu F, Gilks B, et al.: EMSY links the BRCA2 pathway to sporadic breast and ovarian cancer. Cell 2003, 115:523-535.

9. Venkitaraman AR: Cancer susceptibility and the functions of BRCA1 and BRCA2. Cell 2002, 108:171-182.

10. Powell SN, Kachnic LA: Roles of BRCA1 and BRCA2 in homologous recombination, DNA replication fidelity and the cellular response to ionizing radiation. Oncogene 2003, 22:57845791.
11. Jasin M: Homologous repair of DNA damage and tumorigenesis: the BRCA connection. Oncogene 2002, 21:8981-8993.

12. Scully R, Chen J, Plug A, Xiao Y, Weaver D, Feunteun J, Ashley T, Livingston DM: Association of BRCA1 with Rad51 in mitotic and meiotic cells. Cell 1997, 88:265-275.

13. Sharan SK, Morimatsu M, Albrecht U, Lim DS, Regel E, Dinh C, Sands A, Eichele G, Hasty P, Bradley A: Embryonic lethality and radiation hypersensitivity mediated by Rad51 in mice lacking Brca2. Nature 1997, 386:804-810.

14. Xu X, Weaver Z, Linke SP, Li C, Gotay J, Wang XW, Harris CC, Ried T, Deng CX: Centrosome amplification and a defective G2-M cell cycle checkpoint induce genetic instability in BRCA1 exon 11 isoform-deficient cells. Mol Cell 1999, 3:389395.

15. Yuan SS, Lee SY, Chen G, Song M, Tomlinson GE, Lee EY: BRCA2 is required for ionizing radiation-induced assembly of Rad51 complex in vivo. Cancer Res 1999, 59:3547-3551.

16. Chapman MS, Verma IM: Transcriptional activation by BRCA1. Nature 1996, 382:678-679.

17. Monteiro AN, August $\mathrm{A}$, Hanafusa $\mathrm{H}$ : Evidence for a transcriptional activation function of BRCA1 C-terminal region. Proc Natl Acad Sci USA 1996, 93:13595-13599.

18. Milner J, Ponder B, Hughes-Davies L, Seltmann M, Kouzarides T: Transcriptional activation functions in BRCA2. Nature 1997, 386:772-773.

19. Shin S, Verma IM: BRCA2 cooperates with histone acetyltransferases in androgen receptor-mediated transcription. Proc Natl Acad Sci USA 2003, 100:7201-7206.

20. Nordling $M$, Karlsson $P$, Wahlstrom J, Engwall $Y$, Wallgren $A$, Martinsson T: A large deletion disrupts the exon 3 transcription activation domain of the BRCA2 gene in a breast/ovarian cancer family. Cancer Res 1998, 58:1372-1375.

21. Pilch DR, Sedelnikova OA, Redon C, Celeste A, Nussenzweig A Bonner WM: Characteristics of gamma-H2AX foci at DNA double-strand breaks sites. Biochem Cell Biol 2003, 81:123129.

22. Scully R, Chen J, Ochs RL, Keegan K, Hoekstra M, Feunteun J, Livingston DM: Dynamic changes of BRCA1 subnuclear location and phosphorylation state are initiated by DNA damage. Cell 1997, 90:425-435.

23. Maurer-Stroh S, Dickens NJ, Hughes-Davies L, Kouzarides $T$, Eisenhaber F, Ponting CP: The Tudor domain 'Royal Family': Tudor, plant Agenet, Chromo, PWWP and MBT domains. Trends Biochem Sci 2003, 28:69-74.

24. Bochar DA, Wang L, Beniya H, Kinev A, Xue Y, Lane WS, Wang W, Kashanchi $F$, Shiekhattar R: BRCA1 is associated with a human SWI/SNF-related complex: linking chromatin remodeling to breast cancer. Cell 2000, 102:257-265.

25. Marmorstein LY, Kinev AV, Chan GK, Bochar DA, Beniya $H$, Epstein JA, Yen TJ, Shiekhattar R: A human BRCA2 complex containing a structural DNA binding component influences cell cycle progression. Cell 2001, 104:247-257.

26. Bekri S, Adelaide J, Merscher S, Grosgeorge J, Caroli-Bosc F, Perucca-Lostanlen D, Kelley PM, Pebusque MJ, Theillet C, Birnbaum D, Gaudray P: Detailed map of a region commonly amplified at $11 \mathrm{q} 13 \rightarrow \mathrm{q} 14$ in human breast carcinoma. Cytogenet Cell Genet 1997, 79:125-131.

27. Ormandy CJ, Musgrove EA, Hui R, Daly RJ, Sutherland RL: Cyclin D1, EMS1 and 11q13 amplification in breast cancer. Breast Cancer Res Treat 2003, 78:323-335. 